\title{
Understanding the Link between Dementia and Diabetes
}

\author{
Ralph N. Martins $\mathrm{a}^{\mathrm{a}, \mathrm{b}, \mathrm{c}, \mathrm{d}, \mathrm{e}, *}$

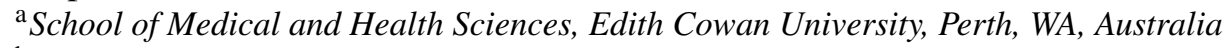 \\ ${ }^{\mathrm{b}}$ Department of Biomedical Sciences, Macquarie University, Sydney, NSW, Australia \\ ${ }^{\mathrm{c}}$ KaRa Institute of Neurological Diseases, Sydney, NSW, Australia \\ ${ }^{\mathrm{d}}$ Australian Alzheimer's Research Foundation Perth, WA, Australia \\ ${ }^{\mathrm{e}}$ School of Psychiatry and Clinical Neurosciences, University of Western Australia, Perth, WA, Australia
}

The concept that type 2 diabetes (T2D)/insulin resistance is associated with dementia risk and pathogenesis has gained momentum over the past few decades. However, there are still gaps in our understanding of the direct/indirect impact of T2D on dementia risk, onset or progression and underlying pathological mechanisms. This link also suggests that therapeutic/ preventative strategies targeting T2D may have benefits for dementia. A number have been evaluated in in vivo animal studies and some are entering clinical trials, however, determining the type of single or combination therapies and when to implement requires further investigation. In addition, more recent evidence also provides new insights and exciting research avenues in exploring the contribution of the Alzheimer's disease (AD) pathogenic amyloid- $\beta$ $(\mathrm{A} \beta)$ and tau proteins to T2D.

These were the topics of a workshop held between 13-15 October 2015, within the picturesque and quiet small town of Westbury, in the central North of Tasmania generously hosted by Dr. Gilbert Stokes. Westbury was the perfect location to assemble a small gathering of Australian and international basic and clinical researchers in the fields of T2D and Neurodegeneration to participate in this workshop. Delegates were asked to contribute to a series of

\footnotetext{
${ }^{*}$ Correspondence to: Professor Ralph Martins, Centre of Excellence in Alzheimer's Disease Research and Care, School of Medical Sciences, Edith Cowan University, 270 Joondalup Drive, Joondalup, WA 6027, Australia. Tel.: +618 63045456; Fax: +618 63045851; E-mail: r.martins@ecu.edu.au.
}

articles on topics which were discussed that make up this mini-forum in the Journal of Alzheimer's Disease.

The article by Sutherland et al. [1] discusses our current understanding of the epidemiological evidence linking T2D with dementia and the importance of continuing this research. Uncertainty still remains concerning the degree of relative risk of T2D to the development of cognitive impairment and dementia and other factors that should be taken into account when estimations of risk are made. As health care, clinical care and environment improve, the epidemiology of both conditions is changing. However, of greater concern is the increasing incidence of T2D among the young. The impact on the development of dementia is not yet known, but as type 2 diabetics are living to an older age, this could significantly impact on prevalence of dementia world-wide. Although there have been population based studies, or metaanalyses to provide a "risk" estimate of T2D on the onset of dementia, demographic, lifestyle, genetic risk factors or premature mortality that could bias estimations are not always taken into account in these studies. A related challenge is teasing out the large number of potential factors related to T2D that may contribute to dementia risk and determining if diabetes is an early risk factor for dementia. The authors suggest a way forward in which in addition to demographics, the incorporation of neuroimaging, clinical pathology, cerebrospinal fluid/blood biomarkers and analysis in large, longitudinal studies are essential in 
providing clarity on the risk that diabetes imposes on developing dementia.

The value of such studies is starting to be recognized. Neuroimaging in particular has provided mechanistic insight into the impact of T2D on dementia and can provide a useful tool for clinical correlation/interventional studies. The article by Moran et al. [2] discusses this further, with a focus on the use of structural and functional MRI and PET brain imaging modalities to assess atrophy, structural networks, functional connectivity, cerebral glucose metabolism and AD-related pathology in diabetics.

There is a great deal of evidence from imaging studies of increased brain atrophy and greater impairment in brain function in type 2 diabetics compared to non-diabetics. However, there is now greater appreciation that cognitive impairment also occurs in type-1 diabetics with more recent studies show similar structural and functional changes in young adults and middle age adults that have type 1 diabetes. However, compared to studies of T2D, there is paucity of data for type 1 diabetes. Further studies are essential to understand better the effects of type 1 diabetes on the brain and the degree to which type 1 diabetes impacts the progression of neurodegeneration.

Neuroimaging has provided insight into the relationship between T2D, brain function and dementia and together with other biomarkers has provided potential associations with AD related pathology in particular determining associations with amyloid plaque deposition and tau accumulation. Although these studies have provided mixed results, there are several cross-sectional studies that have shown stronger associations between T2D and tau accumulation. Longitudinal studies will provide further insight into this relationship and aid in defining at what stage (s) in the progression of pathology the relationship is the strongest. This information is essential in evaluating the benefits of T2D-targeted therapeutic or preventative strategies for $\mathrm{AD}$.

In vitro and in vivo animal studies have provided significant insight into the impact of insulin resistance/ T2D on the accumulation of AD pathology and neuronal functioning. The biological basis is not completely understood but can involve a number of mechanisms that include impaired insulin signaling, inflammatory or oxidative stress that promote metabolic, vascular and neuronal dysfunction. The article by Bharadwaj et al. [3] focuses on the role of T2D in mediating these neurodegenerative signals through the accumulation of $A \beta$ and tau, and also discusses a potential role for another amyloidogenic protein, amylin. Amylin is known to accumulate progressively in the pancreas of T2D patients contributing to $\beta$-cell dysfunction and loss, which results in overt type 1 phenotype at chronic stages of the disease. Amylin can also accumulate in the brain of T2D and $\mathrm{AD}$ patients and contribute to the neurodegenerative process. Bharadwaj and colleagues [3] have proposed that amylin can contribute and enhance the neurodegenerative process through interactions and heterologous cross-seeding, where aggregates of one amyloid protein (e.g., amylin) can promote the aggregation of the other amyloid $(\mathrm{A} \beta)$ protein.

The review discusses mounting evidence that $A \beta$ and tau accumulation can also contribute to a diabetic phenotype. This could occur through impairing insulin signaling in insulin sensitive peripheral organs (i.e., skeletal muscle) or deposition within the $\beta$ islet cells contributing to cell dysfunction. Further, Tau expression in peripheral tissues may be important for maintaining glucose homeostasis. The article presents some recent observations of spliced isoforms of tau in human and mouse pancreatic islets. Together with the seminal findings by Miklossy and colleagues [4] that hyperphosphorylated tau and $A \beta$ accumulate in pancreatic tissue, this suggests that these AD-related proteins may influence insulin secretion and related metabolic pathways. The contribution of $A \beta / T a u$ to $T 2 D$ requires further exploration, but sets up an interesting scenario where AD-targeted therapies could have benefits for T2D.

Publications in the field of lipidomics have increased recently, mostly driven by advances in high pressure liquid chromatography and mass spectrometry technologies. In particular, T2D and AD have received considerable attention in lipidomics studies, due to their prevalence and likely increase in western societies, as a result of rising obesity levels and increases in older populations. In the review by Huynh et al. [5], the greater understanding provided by such lipidomic studies of the relationship between lipid metabolism, T2D and AD is discussed, and how this has brought focus onto some pathological pathways that may link diabetes risk with dementia.

The review details evidence of dysregulation of ceramide, sulfatide, and plasmalogen lipids, with specific links to metabolic pathways and pathogenic changes. In particular, higher ceramide levels and lower levels of plasmalogens appear to be common to both $\mathrm{AD}$ and $\mathrm{T} 2 \mathrm{D}$, and these have been associated with insulin resistance, inflammation, oxidative stress, AD amyloid production, tau phosphorylation and cellular apoptosis. The review also discusses 
evidence of rises in ceramide levels and changes in certain phospholipids that can be detected at preclinical stages of $\mathrm{AD}$; as well as the ratio of phosphatidylcholine to phosphatidylethanolamine and links to obesity, dyslipidemia and T2D, and how this may relate to $\mathrm{AD}$.

The review by Asih et al. [6] discusses how testosterone levels may influence the metabolic abnormalities that have been associated with both $\mathrm{T} 2 \mathrm{D}$ and $\mathrm{AD}$, including insulin resistance, obesity, oxidative stress and chronic inflammation. Impairments in insulin receptor signaling steps in both $\mathrm{T} 2 \mathrm{D}$ and $\mathrm{AD}$ are described, as well as evidence for testosterone influencing insulin-receptor levels and downstream signaling events. Testosterone also downregulates the enzyme BACE1 which is involved in the generation of $A \beta$. While BACE1 is well recognized for its role in $\mathrm{AD}$, it also plays an important role in T2D in that it influences insulin sensitivity. Another link between $\mathrm{AD}$ and diabetes is the fact that insulin degrading enzyme (IDE) is also a major $A \beta-$ degrading enzyme, and testosterone has been shown to modulate expression of IDE (as well as another A $\beta$-degrading enzyme - neprilysin), Similarly, selective androgen receptor modulators can increase IDE levels and activity, as well as neprilysin activity. The increased IDE and neprilysin may reduce both insulin and $A \beta$ levels, possibly reducing pathogenesis of both conditions. The evidence for a bi-directional relationship between glucose metabolism and testosterone levels is discussed, as well as evidence for influences of testosterone levels on lipid metabolism, though in both cases, there are some contradictory findings. There is also discussion concerning the potential preventative therapy of exercise and dietary changes combined with testosterone treatment to reduce risk of both T2D and AD, and the need for more basic research and clinical trials on this topic.

The hyperglycemia of T2D leads to the formation of dicarbonyl compounds such as glyoxal (GO), methylglyoxal (MGO), and 3-deoxyglucosone (3DG MGO), all derived from glucose, and known to be elevated in T2D. These dicarbonyl compounds react with amino acids to form advanced glycation end-products (AGEs), which have been implicated in the pathology and advancement of many chronic and degenerative diseases, including T2D and AD. In T2D, AGEs are thought to contribute to retinopathy, neuropathy, nephropathy and cognitive impairment; whereas in AD, AGEs lead to oxidative stress, chronic inflammation and mitochondrial dysfunction, all of which are important factors in the pathogenesis of AD. The work presented here by Dhananjayan and colleagues [7] describe recent studies of the dicarbonyl MGO, which is known to activate macrophages via the receptor RAGE. The authors show that inflammatory activation of macrophages and microglial cells (using lipopolysaccharide and interferon- $\gamma$ ) results in greater MGO concentrations, thus completing a vicious cycle. This study provides more evidence for inflammatory mechanisms being common to both T2D and $\mathrm{AD}$, as the inflammatory changes caused by higher MGO lead to higher AGE levels, which are linked to hypertension, coronary heart disease, brain ischemia and more advanced T2D, all of which are known to increase the risk of $\mathrm{AD}$.

Tea and various components of tea have been associated with a variety of health benefits, including anti-microbial, anti-inflammatory, anti-tumorigenic, anti-viral, antioxidant and anti-diabetic properties.

Fresh tea leaves comprise approximately 25 to $35 \%$ polyphenolic compounds, and the health benefits have been attributed to these compounds. The review by Fernando and colleagues [8] presents evidence for tea or tea extract consumption reducing the risk of $\mathrm{AD}$, as well as aspects of cardiovascular disease and diabetes. For example, longitudinal and epidemiological studies have shown that 3 or more cups of tea reduces risk of cardiovascular disease and stroke, particularly green tea. The review also summarizes clinical studies which have found that certain polyphenols appear to reduce obesity, possibly by increasing lipid metabolism, though in this case black tea is found to be better than green tea.

Many tea extracts have been studied and results are discussed, with one standing out as being particularly effective - the flavonoid epigallocatechin-3-gallate (EGCG). For example, EGCG has being shown to promote non-amyloidogenic pathways, and EGCG and gallic acid also reduce $A \beta$ aggregation. With respect to diabetes, epidemiological and clinical studies have shown tea consumption reduces risk of T2D. Animal studies support this, as green tea containing EGCG ( $25 \mathrm{mg} / \mathrm{kg}$ body weight) fed to streptozotocininduced diabetic rats has been shown to reduce the rat serum glucose, total cholesterol, triglyceride and LDL-cholesterol levels. Some tea components have been examined with respect to cognition, for example caffeine and L-theanine in tea improve memory, attention, control responsiveness, and other aspects of cognition in clinical studies.

It is clear from the review that although many promising effects of tea consumption can be seen, 
the mechanisms behind these effects have only been partly elucidated, and steps such as tea compound digestion, absorption, transport, and transformation into other compounds are still being investigated.

Furthermore, the results were widely different: consumption of tea has been reported to increase antioxidant activity by anything from 3.5 to $70 \%$, and the quantities of EGCG, epicatechin, and epicatechin gallate in plasma ranged from $5-150 \mathrm{ng} / \mathrm{ml}$, depending on study. This may be due to tea or extract quality, dose, and experimental techniques, However, overall, the review provides evidence of many benefits of tea and tea extract consumption for the abovementioned conditions. By knowing the overlap in metabolic changes that lead to T2D, cardiovascular disease and $\mathrm{AD}$, future studies of tea consumption should investigate a greater number of parameters to help determine benefits for these conditions, and to help determine the mechanisms behind them.

The mini-forum concludes with the potential of lifestyle interventions in reducing the impact of T2D on dementia risk. Callisaya and Nosaka [9] review the evidence for the benefit of exercise on T2D related cognitive impairment and dementia and propose that exercise interventions may have the largest effect on those at high risk of dementia such as those with T2D. The article discusses the number of variables that have to be taken into account (i.e., mode, intensity, frequency) to assess the benefits of exercise but also proposes that multi-modal training programs and eccentric exercise may show promise in offering benefits.

The links now understood to exist between T2D and $\mathrm{AD}$ consolidate the theory that there are common pathogenic mechanisms at play, and although beyond the main scope of the discussion topics of the paper and the reviews here, many of these pathogenic mechanisms are similar to those of cardiovascular disease. Further research into the earliest stages of dysregulation of lipid and glucose metabolism and their associated inflammation and oxidative stress in $\mathrm{T} 2 \mathrm{D}$ and $\mathrm{AD}$ is helping to provide earlier biomarkers of both, and revealing the importance of potential preventative mechanisms - essential in the quest to reduce the incidence of both of these conditions.

\section{REFERENCES}

[1] Sutherland GT, Lim J, Srikanth V, Bruce DG (2017) Epidemiological approaches to understanding the link between type 2 diabetes and dementia. J Alzheimers Dis 59, 393-403.

[2] Moran C, Beare R, Phan T, Starkstein S, Bruce D, Mizrahi R, Srikanth V (2017) Neuroimaging and its relevance to understanding pathways linking diabetes and cognitive dysfunction. J Alzheimers Dis 59, 405-419.

[3] Bharadwaj P, Wijesekara N, Liyanapathirana M, Newsholme P, Ittner L, Fraser P, Verdile G (2017) The link between type 2 diabetes and neurodegeneration: Roles for amyloid- $\beta$, amylin, and tau proteins. J Alzheimers Dis 59, 421-432.

[4] Miklossy J, Qing H, Radenovic A, Kis A, Vileno B, László F, Miller L, Martins RN, Waeber G, Mooser V, Bosman F, Khalili K, Darbinian N, McGeer PL (2010) Beta amyloid and hyperphosphorylated tau deposits in the pancreas in type 2 diabetes. Neurobiol Aging 31, 1503-1515.

[5] Huynh K, Martins RN, Meikle PJ (2017) Lipidomic profiles in diabetes and dementia. J Alzheimers Dis 59, 433-444.

[6] Asih PR, Tegg ML, Sohrabi H, Carruthers M, Gandy SE, Saad F, Verdile G, Ittner LM, Martins RN (2017) Multiple mechanisms linking type 2 diabetes and Alzheimer's disease: Testosterone as a modifier. J Alzheimers Dis 59, 445-466.

[7] Dhananjayan K, Gunawardena D, Hearn N, Sonntag T, Moran C, Gyengesi E, Srikanth V, Münch G (2017) Activation of macrophages and microglia by interferon- $\gamma$ and lipopolysaccharide increases methylglyoxal production: A new mechanism in the development of vascular complications and cognitive decline in type 2 diabetes mellitus? J Alzheimers Dis 59, 467-479.

[8] Fernando WMADB, Somaratne G, Goozee KG, Williams S, Singh H, Martins RN (2017) Diabetes and Alzheimer's disease: Can tea phytochemicals play a role in prevention? J Alzheimers Dis 59, 481-501.

[9] Callisaya M, Nosaka K (2017) Effects of exercise on type 2 diabetes mellitus-related cognitive impairment and dementia. J Alzheimers Dis 59, 503-513. 